\title{
Impaired lipopolysaccharide responsiveness of cord blood mononuclear cells and the risk of asthma: a longitudinal study
}

\author{
Michael R. Goldberg ${ }^{1,2}$, Arnon Elizur ${ }^{1,2}$, Noemi Luknar-Gabor', Penina Koch', Joseph Tovbin ${ }^{3}$ and Yitzhak Katz ${ }^{1,2}$
}

BACKGROUND: We previously demonstrated that the proliferative response to lipopolysaccharide (LPS) in cord blood mononuclear cells (CBMCs) is negatively correlated with the induced expression of interleukin (IL)-4. Our aim, therefore, was to examine whether an impaired cellular response to LPS in infancy is associated with the risk for asthma.

METHODS: In a prospective cohort study, the relationship between the CBMC response to LPS and the risk of atopy and wheezing after the age of $4 \mathrm{y}$ was evaluated.

RESULTS: LPS-induced CBMC proliferative responses varied markedly among the 102 infants studied (range, one- to fivefold increase over cells with diluent alone). Ninety-five infants (93\%) were followed longitudinally. A higher CBMC proliferative response to LPS was noted in offspring born to nonatopic parents compared with those with at least one atopic parent $(P=0.008)$. Using a proliferative index cutoff of 2 separated patients into high and low induced IL-4 mRNA responders $(P=0.001)$. Significantly more children who never wheezed had a greater than twofold LPS-induced CBMC proliferative response compared to those with persistent atopic wheezing $(P=0.046)$. CONCLUSION: These results demonstrate that CBMC proliferative responses to LPS is impaired in infants born to atopic parents and may be a risk factor for asthma later in life.

들 pidemiological studies and experimental models sugexposure to innate stimuli, such as endotoxin, can modulate the susceptibility to atopy in humans (1-3) Increasing the complexity of these findings is that exposure to lipopolysaccharide (LPS) may be protective against the development of atopy, but only in the context of each individual's genotype (4). Mouse model studies demonstrate that LPS can have a protective effect against atopy and allergy (5-7).

For an environmental stimulus to exert an immunomodulatory effect, as suggested by these epidemiological and molecular studies, it is likely to affect cells of the immune system early in development, yielding changes in the cytokines expressed as well as in proliferation $(8,9)$. For example, in a longitudinal study, increased exposure at age 2-3 mo to household endotoxin was associated with reduced allergen-induced production of the TH2 cytokine IL-13, in children at risk for allergy and asthma (10). Consistent with this report, monocytes from children with atopy demonstrated impaired LPS-induced TLR4-signalling (11). With regard to the proliferative response to environmental stimulants, we have shown that cord blood mononuclear cells (CBMCs) differ from peripheral blood mononuclear cells (PBMCs), suggesting an important role in the modulation of the immune system. For example, human CBMCs have an increased proliferative response to LPS relative to their respective maternal PBMCs (12). Furthermore, a high proliferative response to LPS in CBMCs correlated with a change from a Th2- to Th1-induced cytokine expression profile (13), which may help protect against a universal skewing of initial T-cell responses toward a Th2 cytokine profile (14). Thus, the CBMC proliferative response to LPS, partly because of its heterogeneity, may be a good surrogate marker of an infant's subsequent immune predisposition to allergy.

The above-mentioned studies evaluated independently, either the clinical response to environmental endotoxin exposure or the cellular response to LPS. In one study of a high-risk cohort with a familial history of allergic disease, early-onset wheeze up to the age of $2 \mathrm{y}$ correlated with an enhanced CBMC cytokine response to LPS (15). Many children who wheeze before the age of $3 \mathrm{y}$, however, are not likely to develop asthma (16). The current analysis examines in a population-based cohort the LPS-induced proliferative responses in CBMCs and their association with wheezing and markers of atopy, later in life.

\section{RESULTS}

\section{LPS-Induced Proliferative Effect on CBMCs}

Overall, 102 children entered the study; most were born by cesarean section (74.5\%) and were males (54.9\%) (Table 1). The mode of delivery and gender, however, did not affect the CBMC proliferative response to LPS or phytohemagglutinin (PHA) (Figure 1a,b). We previously demonstrated that the proliferative response to LPS in CBMCs is negatively correlated with the expression of IL-4 (11). Further analysis of those data revealed that using a proliferative index cutoff of 2 separated these patients into high and low induced IL-4 mRNA responders and may therefore differentiate between patients with high and low atopic risk $(P=0.001)$ (Figure 2)

\footnotetext{
The first two authors contributed equally to this work.

IInstitute of Allergy and Immunology, Assaf Harofeh Medical Center, Zerifin, Israel; ${ }^{2}$ Department of Pediatrics, Sackler School of Medicine, Tel-Aviv University, Tel Aviv, Israel;

${ }^{3}$ Department of Obstetrics and Gynecology, Assaf Harofeh Medical Center, Zerifin, Israel. Correspondence: Michael R. Goldberg (goldbergsm@yahoo.com)
} 
Table 1. Characteristics of the study population

\begin{tabular}{|c|c|c|c|c|}
\hline & \multicolumn{2}{|c|}{ Parameter } & Number & Percentage \\
\hline \multirow[t]{11}{*}{ Child } & \multicolumn{2}{|l|}{ Male } & $56 / 102$ & 54.9 \\
\hline & \multicolumn{2}{|c|}{ C-section delivery } & $76 / 102$ & 74.5 \\
\hline & \multirow{3}{*}{$\begin{array}{l}\text { Age at final } \\
\text { follow-up }\end{array}$} & $<4$ y old & $4 / 95$ & 4.2 \\
\hline & & $4-6$ y old & $48 / 95$ & 50.5 \\
\hline & & $\geq 6$ y old & $43 / 95$ & 45.3 \\
\hline & \multirow[t]{4}{*}{ Wheezing $^{\mathrm{a}}$} & No & $40 / 91$ & 44 \\
\hline & & Yes & $51 / 91$ & 56 \\
\hline & & Transient & $30 / 91$ & 33 \\
\hline & & Persistent & $21 / 91$ & 23 \\
\hline & \multicolumn{2}{|c|}{ Atopic dermatitis (MD diagnosis) } & $4 / 95$ & 4.2 \\
\hline & \multicolumn{2}{|l|}{ Positive SPT } & $5 / 46$ & 10.9 \\
\hline \multirow[t]{9}{*}{ Parent } & \multirow{3}{*}{$\begin{array}{l}\text { Allergy or } \\
\text { asthma (MD } \\
\text { diagnosis) }\end{array}$} & Maternal & $18 / 95$ & 18.9 \\
\hline & & Paternal & $7 / 91$ & 7.7 \\
\hline & & $\begin{array}{l}\text { In at least one } \\
\text { parent }^{\mathrm{b}}\end{array}$ & $24 / 92$ & 26.1 \\
\hline & \multirow[t]{3}{*}{ Positive SPT } & Maternal & $16 / 48$ & 33.3 \\
\hline & & Paternal & $16 / 45$ & 35.6 \\
\hline & & $\begin{array}{l}\text { In at least one } \\
\text { parent }^{\text {b }}\end{array}$ & $26 / 46$ & $56 / 5$ \\
\hline & \multirow[t]{3}{*}{ Elevated $\lg \mathrm{E}^{\mathrm{c}}$} & Maternal & $3 / 23$ & 13 \\
\hline & & Paternal & $10 / 21$ & 47.6 \\
\hline & & $\begin{array}{l}\text { In at least one } \\
\text { parent }\end{array}$ & $13 / 22$ & 59.1 \\
\hline
\end{tabular}

C-section, cesarean section; SPT, skin prick test.

a Of those who were $\geq 4 y$ at time of clinical evaluation. blf only one of the parents was tested and found negative, this family was not included in the denominator. ${ }^{9} \mathrm{~g} E>100$ $\mathrm{IU} / \mathrm{ml}$, as per the manufacturer's guidelines.

(15). Of the 95 children clinically followed, 26 (27.4\%) had a greater than or equal to twofold proliferative index increase, $55(57.9 \%)$ had a proliferative index between 1 and 2, and $14(14.7 \%)$ had a proliferative index $\leq 1$ in response to LPS, whereas the response to PHA was more robust (Figure $\mathbf{1 b}$ ).

\section{Children's Wheezing Phenotypes and Parental Atopic Status}

The ages of children at clinical evaluation and their atopic status are shown in Table 1. Four infants were lost to followup after the first year and were not included in subsequent analysis of wheezing. Of the remaining 91 infants, wheezing before 3 y was reported in 56\% (51/91), but only 23\% (21/91) had persistent wheezing. No wheezing was reported in $44 \%$ (40/91) of the children. The individual maternal and paternal atopic status as defined by a physician diagnosis of allergy or asthma, a positive skin prick test (SPT), or an elevated $\operatorname{IgE}$ is presented in Table 1. Whereas all mothers with an MD diagnosis of allergic rhinitis (AR) who were skin tested had a positive SPT, maternal MD diagnosis of asthma and paternal MD diagnosis of AR or asthma were reported even in parents with a negative SPT (data not shown). In addition, only 9 of the 16 (56.3\%) mothers and 3 of the 16 (18.8\%) fathers who had a positive SPT were given an MD diagnosis of an allergic disease (data not shown). a
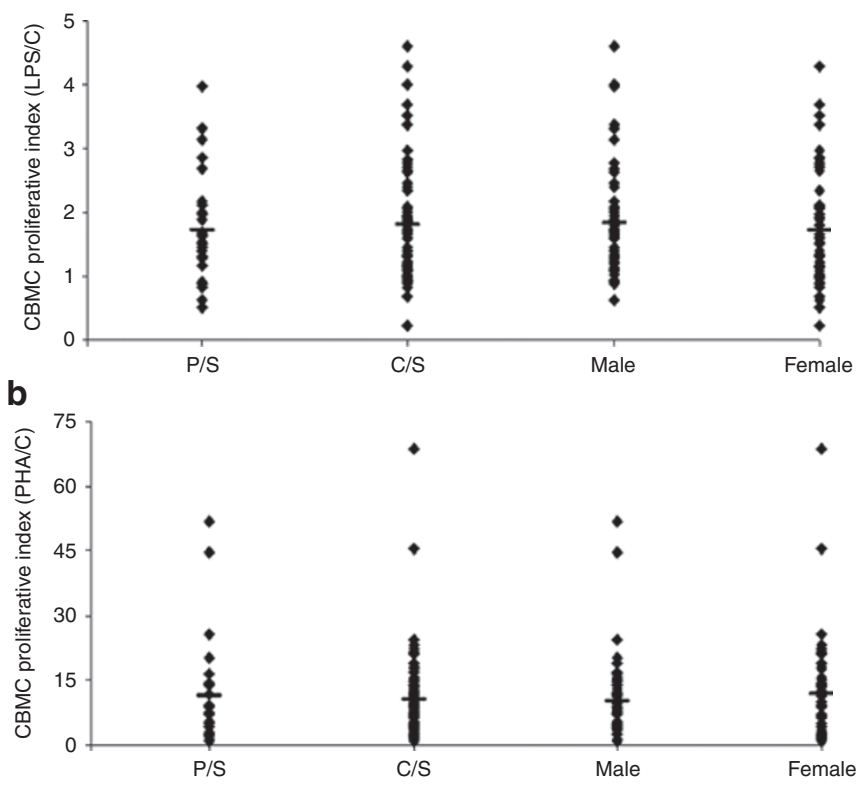

Figure 1. Proliferative responses of CBMCs to LPS. The proliferative index was obtained by dividing the CBMC responses to (a) LPS $(10 \mu \mathrm{g} / \mathrm{ml})$ and (b) PHA $(5 \mu \mathrm{g} / \mathrm{ml})$ by their respective controls. The LPS- or PHA-induced $\mathrm{CBMC}$ response was compared on the basis of mode of delivery and gender of infants. Each dot represents an average of each individual's response performed in triplicate. No statistical differences were noted between the different groups. C/S, cesarean section; CBMC, cord blood mononuclear cell; LPS, lipoplysaccharide; PHA, phytohemagglutinin; P/S, partum spontaneous.

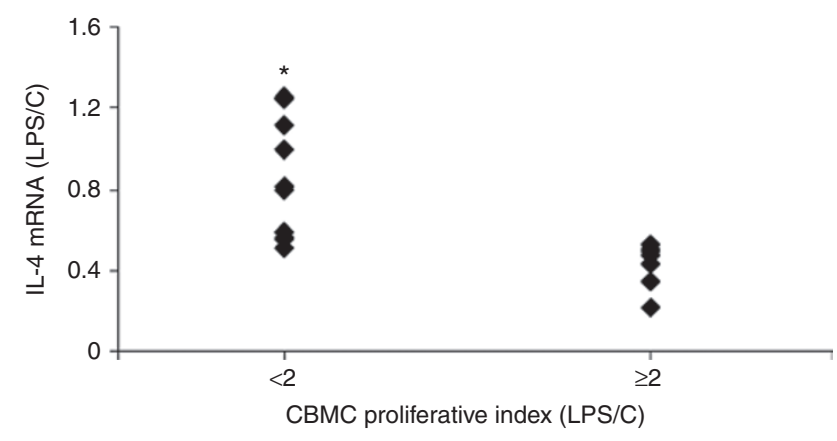

Figure 2. A proliferative index cutoff of two separate high and low $\mathrm{IL}-4$ responders. The correlation between the LPS-induced $(10 \mu \mathrm{g} / \mathrm{ml})$ proliferative response and the induced IL-4 mRNA expression levels was previously described (11). The induced IL-4 mRNA levels were significantly higher in patients with CBMC proliferative index $<2$ as compared with the levels in patients with a proliferative index $\geq 2(0.84 \pm 0.29 \mathrm{vs}$. $0.43 \pm 0.11$, respectively). ${ }^{*} P=0.001$ for proliferative index $<2$ vs. proliferative index $\geq 2$. CBMC, cord blood mononuclear cell; IL-4, interleukin 4; LPS, lipopolysaccharide.

\section{Parental Atopy and CBMC Proliferative Response}

We next evaluated whether an association existed between an infant's CBMC response to LPS and parental atopy. The CBMC proliferative response to LPS of an infant born to at least one atopic parent was significantly lower compared to infants born to nonatopic parents (median 1.2, range $0.52-2.68$ vs. 1.68 , range 0.9 - to 4.3 -fold increase over control, respectively, $P=0.008$ ), whereas an MD-diagnosed parental allergy or elevated serum IgE levels did not demonstrate a similar 


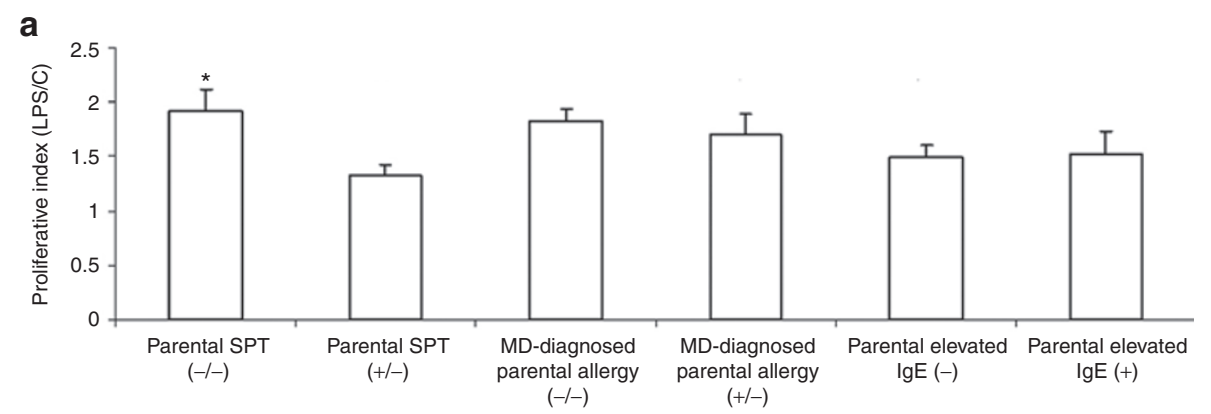

b

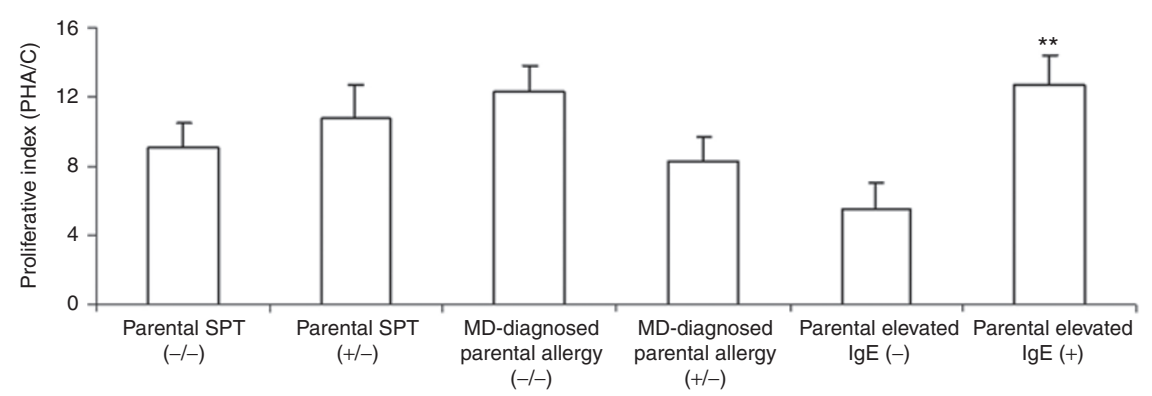

Figure 3. Relationship between CBMC-induced proliferative responses and parental atopy. CBMCs were isolated and stimulated either LPS (10 $\mu \mathrm{g} / \mathrm{ml}$ ) or PHA $(5 \mu \mathrm{g} / \mathrm{ml})$, as described in the Methods. The association between the atopic status of the parents, as determined by parental SPT, MD diagnosis of allergic disease (parental allergy), and parental total serum IgE and the proliferative index of CBMCs in response to (a) LPS and (b) PHA is presented. Parental SPT $(-/-)=$ both mother and father had a negative SPT. Parental SPT $(+/-)=$ either the mother or father had a positive SPT. The LPS response in CBMCs with a parent with a positive SPT was significantly lower as compared with that in CBMCs from parents who were both SPT negative. ${ }^{*} P<0.01$ for parental SPT (+/-) vs. parental SPT (-/-). In addition, a greater stimulative response to PHA was significantly associated with an elevated parental lgE. ${ }^{* *} P$ $<0.05$ for parental elevated IgE (+) vs. parental elevated IgE (-). Bars represent mean and \pm SE measurements. CBMCs, cord blood mononuclear cells; LPS, lipopolysaccharide; PHA, phytohemagglutinin; SPT, skin prick test.

association (Figure 3a). In contrast, the proliferative response to a polyclonal T-cell activator PHA did not demonstrate a significant correlation with parental positive SPT (Figure 3b). Interestingly, an elevated parental IgE level was significantly associated with a higher CBMC proliferative response to PHA (median 12.6, range 1.94-22.3 vs. 4.1, range 1.4- to 11.9-fold increase over control, respectively, $P=0.034$ ), but an opposite trend was noted when parental allergy was used as the parameter (Figure $3 \mathbf{b}$ ). The CBMC proliferative response to LPS was significantly lower in children who returned for SPTs compared to those who were not tested (median 1.4, range $0.52-4.3$ vs. 1.8 , range $0.59-4.6, P=0.023$ ). The PHA response was not significantly different between the two groups (data not shown). Thus, if there was a bias for those with an allergic background to return for testing, it is consistent with our results that a lower LPS-induced proliferative response correlates with a positive SPT in the parent, as demonstrated in Figure 3a.

\section{Childhood Wheezing and CBMC Proliferative Response}

We next assessed whether a reduced proliferative LPS response in infancy was associated longitudinally with childhood wheezing. For the analyses of the risk of asthma in children, groups for comparison were defined as follows: no wheezing, children $\geq 4$ y who never wheezed; transient wheezing, children $\geq 4$ y who wheezed only before the age of $3 \mathrm{y}$; persistent wheezing + atopy, children $\geq 4$ y with persistent wheezing and evidence of parental or self atopy. A proliferative cutoff

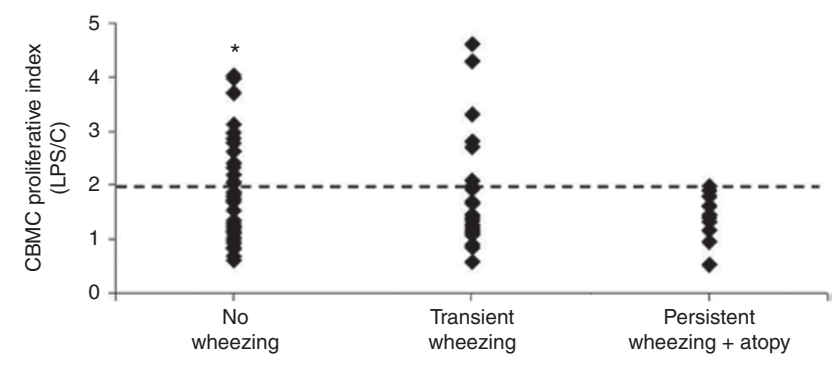

Figure 4. Decreased CBMC proliferative response to LPS is associated with increased risk for asthma in children. The association between various wheezing phenotypes in the children and the LPS-induced CBMC proliferative index was examined. A $\chi^{2}$-test was used to compare the percentage of patients whose CBMC LPS-induced proliferative index was $\geq 2$ in the non-wheezing group vs. those with high-risk wheezing. Dotted bar delineates patients with a proliferative index $\geq 2$ (above dotted line) vs. a proliferative index $<2$ (below line). No wheezing, $n=40$; transient wheezing, $n=30$; persistent wheezing + atopy, $n=11$. ${ }^{*} P<0.05$ for patients with no wheezing vs. those with persistent wheezing + atopy. CBMC, cord blood mononuclear cell; LPS, lipopolysaccharide.

of 2 was used because this significantly separated low and high responders for IL-4 expression (Figure 2). Of particular interest, none of the children with persistent wheezing and an atopic background $(n=11)$, had a CBMC proliferative index $\geq 2$ in response to LPS (Figure 4). In contrast, 32.5\% (13/40) of children who never wheezed had a CBMC proliferative index $\geq 2(P=0.046)$. No association was found between the CBMC proliferative response to LPS or PHA and the occurrence of wheezing isolated to the years before the age of three (transient 


\section{Articles | Goldberget al.}

wheezing). Among the group of children with persistent wheezing including those without evidence of atopy, only $23.8 \%(5 / 21)$ had CBMC proliferative index $\geq 2$, but statistical significance was not met (data not shown). No association was found between CBMC proliferation in response to PHA and any of the wheezing phenotypes in these children (data not shown).

\section{DISCUSSION}

This study using LPS as a surrogate activator of the innate immune system demonstrates that the CBMC response to LPS is influenced by an individual's familial atopic predisposition and correlates with the risk of developing asthma. Offspring born to nonatopic parents had a greater proliferative response of their CBMCs to LPS compared to those with at least one atopic parent. Furthermore, none of the children with persistent wheezing and evidence of atopy had a robust proliferative response to LPS. To our knowledge, this is the first study that uses a longitudinal follow-up to establish the association between CBMC responses to LPS in early life and the risk for asthma later in childhood.

We emphasized persistent wheezing before and after the age of $3 \mathrm{y}$ as one of the main outcome measures because only approximately $40 \%$ of children wheezing in the first $3 \mathrm{y}$ of life will continue to wheeze up to the age of 6 and be at risk for asthma (16). Furthermore, we followed all children to an age of $4 \mathrm{y}$ or more to ensure the validity of our wheezing classifications. Risk factors for asthma in children who wheeze in the first $3 \mathrm{y}$ of life include maternal asthma and positive SPT and atopy in a child $(16,17)$. These parameters were used to construct an asthma predictive index for wheezing children (18). Perhaps not surprisingly, therefore, when we analyzed children with persistent wheezing who had the additional criteria that paralleled the asthma predictive index (18), a significantly lower LPS-proliferative response was noted.

We found associations between the CBMC proliferative response and parental atopy, only when the latter was defined objectively (SPT and total serum IgE). We have previously shown that parental sensitization to allergens, rather than a physician-made diagnosis, confers a risk for asthma (17). Others similarly noted that many patients underreport their respiratory symptoms to general practitioners, thus contributing to an underdiagnosis of asthma (19). Furthermore, in our study, parents with no subjective evidence of atopic symptoms had a positive SPT or elevated IgE, suggesting they may still carry hereditary risk for atopy. Finally, elevated parental serum $\mathrm{IgE}$ was found to be associated with an increased CBMC proliferative response to PHA, the latter perhaps related to a reduced regulatory $\mathrm{T}$-cell suppressive effect (20). The opposite trend was noted when MD-diagnosed allergy was used.

The protective effect of exposure to stables and farm milk in early life was initially demonstrated in several cross-sectional studies (2, for review see 21 ). This protection might be mediated through modulation of the Th1/Th2 balance in the developing immune system, perhaps via an effect on regulatory $\mathrm{T}$ cells, by bacterial products such as LPS $(22,23)$. Indeed, cord blood regulatory $\mathrm{T}$ cell number and function were increased in infants exposed to farm environments during pregnancy, and their function was impaired in infants to atopic mothers $(8,20)$. These investigators did not find a significant effect on the CBMC proliferative response; however, their assay differed from ours in the use of a shorter stimulation time and a lower dose of LPS. In addition, their definition of atopy was based on an MD diagnosis. Other studies support the role of LPS in modulating atopic diseases and asthma by demonstrating that polymorphisms in CD14 and TLR-4, which form part of the LPS-TLR signaling complex, correlated with the presence of atopy (1,24-27). In one longitudinal study of a highrisk cohort, an enhanced CBMC cytokine response to LPS was associated with nonatopic wheezing at the age of 2 y (15). Our study differs in that we analyzed the CBMC proliferative response of infants derived from the general population vs. from a high-risk cohort. Furthermore, our findings relate to infants with persistent atopic wheeze, who are at higher risk to develop asthma (18).

The design of this study depended on the willingness of families to come for evaluation in a hospital setting. Indeed, SPT was performed in only $52.8 \%$ of parents, and $27 \%$ had IgE measurements evaluated. This can produce a bias toward a more atopic population because subjects with allergies are more likely to continue to follow-up. However, the rate of SPT positivity in our parental population is similar to prior descriptions both in Israel and worldwide, and the rate of reported allergic diseases was not higher than expected $(28,29)$. Furthermore, the frequency of transient vs. persistent wheezing in the children we followed matches the descriptions noted by Martinez et al. (16). Regardless, even if the study population was biased toward atopic individuals, our results would be consistent because those who did not arrive for evaluation tended to have both a higher proliferative response to LPS and less wheezing.

In summary, this study demonstrates that parental atopy, as determined by positive SPT, is significantly associated with a lower CBMC proliferative response to LPS. This reduced response, which has been previously shown to be associated with a shift from Th1- to TH2-related cytokine secretion (13), correlates with increased risk for asthma in children. These data should lead to further mechanistic studies on the role of Toll-like receptor agonists in modulating the Th1/Th2 balance in utero or early in life.

\section{METHODS}

\section{Study Design}

This study was approved by the Helsinki committee at the Assaf Harofeh Medical Center, and informed consent was obtained from all study participants. All infants included in the study were born fullterm. Infants with signs of infection such as poor suck, listlessness, breathing difficulty, decreased or elevated temperature, and changes in skin color, were excluded from our analysis. A total of 102 children, 88 singletons and 7 twins, from 95 families entered the study. Study participants were generally recruited during the first $2 \mathrm{~d}$ of the week to allow completion of the proliferative assay, which lasted $72 \mathrm{~h}$, on a weekday. Because elective cesarean sections were generally performed on these days, many of the infants in this study were born via cesarean section. As previously noted (12), however, and confirmed in the larger series presented here, there were no statistical differences 
between the proliferative responses from cells derived from vaginal or cesarean section (Figure 1). The proliferative assay was begun on the same day as that of cord blood collection.

Follow-up included a telephone contact during the first year of life and again after the age of $4 \mathrm{y}$. A questionnaire, based on the International Study of Asthma and Allergies in Childhood (ISAAC) questionnaire (30), that queried for an MD diagnosis of asthma, AR or atopic dermatitis in the parent, and atopic dermatitis or history of wheezing before and after the age of $3 \mathrm{y}$ in the infant was completed. Ninety-five of the 102 children (93\%) were followed, using the questionnaire during the study period ( 83 singletons and 6 twins), and included in the analysis of the CBMC response to LPS, which was used as a predictor of wheezing. The remaining seven children were lost to follow-up. Children were invited for a clinic visit with their parents when they were $1 \mathrm{y}$ old and again after the age of $3 \mathrm{y}$, and SPT and IgE measurements were performed. Overall, SPT was performed in parents from $47(52.8 \%)$ families and IgE was measured in parents from 24 (27\%) families.

\section{Skin Prick Test and IgE Measurement}

Parents and children underwent SPT to allergens (ALK-Abelló, Port Washington, NY) commonly exposed to in their environment, including Dermatophagoides farinae and Dermatophagoides petronyssinus $(5,000 \mathrm{AU} / \mathrm{ml})$, olive tree $(1: 20 \mathrm{w} / \mathrm{v})$, cypress tree $(1: 20 \mathrm{w} / \mathrm{v})$, pecan tree $(1: 20 \mathrm{w} / \mathrm{v})$, perennial rye $(100,000 \mathrm{BAU} / \mathrm{ml})$, mugwort $(1: 20)$, pellitory $(1: 20 \mathrm{w} / \mathrm{v})$, Alternaria $(1: 10 \mathrm{w} / \mathrm{v})$, cat hair $(5000 \mathrm{BAU} / \mathrm{ml})$, cat pelt $(10,000 \mathrm{BAU} / \mathrm{ml})$, and positive control (histamine $1 \mathrm{mg} / \mathrm{ml}$ ). An SPT was considered positive if there was a wheal $\geq 3 \mathrm{~mm}$ in response to one or more of the allergens. Total serum IgE was measured using an immunometric assay, Immulite 2000, (Siemens, Tarrytown, NY) and was considered elevated if it was $>100 \mathrm{IU} / \mathrm{ml}$, as per the manufacturer's guidelines.

\section{Isolation and Proliferation Assays of CBMCs}

CBMC responses to LPS and isolation of total mRNA were performed as previously described $(12,13)$. Briefly, cord blood was obtained from healthy infants with uncomplicated deliveries (either spontaneous or elective cesarean sections) by catheterization of the umbilical vein with an 18-gauge needle and placed into vacutainers containing EDTA. CBMCs were isolated after a Ficoll-Paque (Amersham Biosciences, Uppsala, Sweden) centrifugation and washed twice with RPMI (Biological Industries, Beit Haemek, Israel). Neonatal CBMCs were treated with lysis buffer $(155 \mathrm{mmol} / \mathrm{l}$ ammonium chloride, $10 \mathrm{mmol} / \mathrm{l}$ potassium carbonate, and $0.1 \mathrm{mmol} / \mathrm{l} \mathrm{EDTA}$ ) for $5 \mathrm{~min}$ and then washed twice with RPMI and resuspended in RPMI containing 5\% fetal calf serum (Biological Industries). For the proliferation assay, $2 \times 10^{5}$ CBMCs were seeded in a U-bottom 96-well plate (Costar, Corning, $\mathrm{NY}$ ) in a 100- $\mu$ l volume under the following conditions: medium alone (RPMI containing 5\% fetal calf serum); stimulated with PHA, $5 \mu \mathrm{g} / \mathrm{ml}$ (Sigma, St. Louis, MO); or stimulated with LPS $(10 \mu \mathrm{g} / \mathrm{ml}$, Escherichia coli serotype 0111:B4, Sigma). After $48 \mathrm{~h}$ in culture, cells were pulsed for $16 \mathrm{~h}$ with tritiated thymidine $(1 \mu$ Curie per well, Amersham Biosciences), and then harvested. Filters were placed in Quicksafe A (Zinsser Analytic, Maidenhead, UK) and counted in a LKB liquid scintillation counter. Each point was performed in triplicate. Background counts determined from wells without cells were subtracted from the data. A proliferative index was defined as the proliferative response to LPS or PHA divided by the proliferative response of cells in culture medium alone (RPMI containing 5\% fetal calf serum).

\section{IL-4 Expression Profile}

Total mRNA was isolated from CBMCs after culture in LPS for $72 \mathrm{~h}$ using TRI Reagent (Sigma, Rehovot, Israel), according to the manufacturer's instructions. Our choice of $72 \mathrm{~h}$ was based on our initial experiments demonstrating the optimal proliferative response in CBMCs at this time. Reverse transcription was performed using the Reverse-iT First-Strand Synthesis Kit (ABgene, Surrey, UK). Amplification of IL-4 and GAPDH cDNA by real-time PCR used LightCycler FastStart DNA Master SYBR Green I (Roche, Mannheim, Germany). The primer pairs used for amplification of IL-4 were
5- gTTgACCgTAACAgACATC and 3- ACgTACTCTggTTggC, and for amplification of GAPDH, 5- TCggAgTCAACggATTT and 3- CCACgACgTACTCAgC. The housekeeping gene GAPDH was used as an internal control for normalization of mRNA levels. Results were compared to a standard curve derived from real-time PCR of serial dilutions of cDNA utilizing IL-4-specific primers. A stimulation index was determined as the relative amounts of specific mRNA generated for each cytokine after LPS treatment compared with cells in medium alone.

\section{Statistical Analyses}

Analyses were performed using SPSS 16.0 for Windows (SPSS, Chicago, IL). LPS and PHA proliferation were not normally distributed; therefore, the Mann-Whitney nonparametric test was used for analysis. Values are expressed as means \pm SD unless otherwise stated. Chi-square analysis with Fisher's exact test was used to analyze the relationship between categorical variables, when comparing children who never wheezed and those with persistent wheezing plus atopy. Values with a $P<0.05$ were chosen to be significant.

\section{ACKNOWLEDGMENTS}

We acknowledge Ora Soffrin and Amalya Dror for their help in obtaining blood samples for this study.

\section{STATEMENT OF FINANCIAL SUPPORT}

Funding for this research was provided by the Atzlajá-Flia. Feldman Fund. M.R.G. is funded by a Kamea grant by the Ministry of Health.

Disclosure: The authors declare no conflict of interest.

\section{REFERENCES}

1. Sengler C, Haider A, Sommerfeld C, et al.; German Multicenter Allergy Study Group. Evaluation of the CD14 C-159 T polymorphism in the German Multicenter Allergy Study cohort. Clin Exp Allergy 2003;33:166-9.

2. Braun-Fahrländer C, Riedler J, Herz U, et al.; Allergy and Endotoxin Study Team. Environmental exposure to endotoxin and its relation to asthma in school-age children. N Engl J Med 2002;347:869-77.

3. Douwes J, van Strien R, Doekes G, et al. Does early indoor microbial exposure reduce the risk of asthma? The Prevention and Incidence of Asthma and Mite Allergy birth cohort study. J Allergy Clin Immunol 2006;117:1067-73.

4. Simpson A, Martinez FD. The role of lipopolysaccharide in the development of atopy in humans. Clin Exp Allergy 2010;40:209-23.

5. Bashir ME, Louie S, Shi HN, Nagler-Anderson C. Toll-like receptor 4 signaling by intestinal microbes influences susceptibility to food allergy. J Immunol 2004;172:6978-87.

6. Wang Y, McCusker C. Neonatal exposure with LPS and/or allergen prevents experimental allergic airways disease: development of tolerance using environmental antigens. J Allergy Clin Immunol 2006;118:143-51.

7. Gerhold K, Avagyan A, Seib C, et al. Prenatal initiation of endotoxin airway exposure prevents subsequent allergen-induced sensitization and airway inflammation in mice. J Allergy Clin Immunol 2006;118:666-73.

8. Schaub B, Liu J, Höppler S, et al. Maternal farm exposure modulates neonatal immune mechanisms through regulatory T cells. J Allergy Clin Immunol 2009;123:774-82.e5.

9. Wood RA, Bloomberg GR, Kattan M, et al. Relationships among environmental exposures, cord blood cytokine responses, allergy, and wheeze at 1 year of age in an inner-city birth cohort (Urban Environment and Childhood Asthma study). J Allergy Clin Immunol 2011;127:913-9.e1-6.

10. Abraham JH, Finn PW, Milton DK, Ryan LM, Perkins DL, Gold DR. Infant home endotoxin is associated with reduced allergen-stimulated lymphocyte proliferation and IL-13 production in childhood. J Allergy Clin Immunol 2005;116:431-7.

11. Préfontaine D, Banville-Langelier AA, Fiset PO, et al. Children with atopic histories exhibit impaired lipopolysaccharide-induced Toll-like receptor- 4 signalling in peripheral monocytes. Clin Exp Allergy 2010;40:1648-57.

12. Goldberg MR, Luknar-Gabor N, Zadik-Mnuhin G, Koch P, Tovbin J, Katz Y. Synergy between LPS and immobilized anti-human CD3epsilon $\mathrm{mAb}$ for activation of cord blood CD3+ T cells. Int Immunol 2007;19:99-103. 
13. Goldberg MR, Nadiv O, Luknar-Gabor N, Zadik-Mnuhin G, Tovbin J, Katz Y. Correlation of Th1-type cytokine expression and induced proliferation to lipopolysaccharide. Am J Respir Cell Mol Biol 2008;38:733-7.

14. Prescott SL, Macaubas C, Holt BJ, et al. Transplacental priming of the human immune system to environmental allergens: universal skewing of initial T cell responses toward the Th2 cytokine profile. J Immunol 1998;160:4730-7.

15. Quah PL, Kuo IC, Huang CH, Shek LP, Lee BW, Chua KY. Early onset wheeze associated with enhanced combined IL-1ß, IL-6, and IL-12/IL23p40 in LPS-stimulated cord blood mononuclear cells. Clin Exp Allergy 2011;41:970-8.

16. Martinez FD, Wright AL, Taussig LM, Holberg CJ, Halonen M, Morgan WJ. Asthma and wheezing in the first six years of life. The Group Health Medical Associates. N Engl J Med 1995;332:133-8.

17. Elizur A, Pollack N, Boslaugh SE, Kannai Y, Katz Y. Maternal positive skin prick test results and asthma prediction after early childhood wheezing. Ann Allergy Asthma Immunol 2007;98:540-5.

18. Castro-Rodríguez JA, Holberg CJ, Wright AL, Martinez FD. A clinical index to define risk of asthma in young children with recurrent wheezing. Am J Respir Crit Care Med 2000;162(4 Pt 1):1403-6.

19. van Schayck CP, van Der Heijden FM, van Den Boom G, Tirimanna PR, van Herwaarden CL. Underdiagnosis of asthma: is the doctor or the patient to blame? The DIMCA project. Thorax 2000;55:562-5.

20. Schaub B, Liu J, Höppler S, et al. Impairment of T-regulatory cells in cord blood of atopic mothers. J Allergy Clin Immunol 2008;121:1491-9, 1499. e1-13.

21. von Mutius E, Vercelli D. Farm living: effects on childhood asthma and allergy. Nat Rev Immunol 2010;10:861-8.
22. Kapsenberg ML, Jansen HM, Bos JD, Wierenga EA. Role of type 1 and type 2 T helper cells in allergic diseases. Curr Opin Immunol 1992;4:788-93.

23. Sakaguchi S, Ono M, Setoguchi R, et al. Foxp3+ CD25+ CD4+ natural regulatory $\mathrm{T}$ cells in dominant self-tolerance and autoimmune disease. Immunol Rev 2006;212:8-27.

24. Reece P, Thanendran A, Crawford L, et al. Maternal allergy modulates cord blood hematopoietic progenitor Toll-like receptor expression and function. J Allergy Clin Immunol 2011;127:447-53.

25. Koppelman GH, Reijmerink NE, Colin Stine O, et al. Association of a promoter polymorphism of the CD14 gene and atopy. Am J Respir Crit Care Med 2001;163:965-9.

26. O’Donnell AR, Toelle BG, Marks GB, et al. Age-specific relationship between CD14 and atopy in a cohort assessed from age 8 to 25 years. Am J Respir Crit Care Med 2004;169:615-22.

27. Fagerås Böttcher M, Hmani-Aifa M, Lindström A, et al. A TLR4 polymorphism is associated with asthma and reduced lipopolysaccharide-induced interleukin-12(p70) responses in Swedish children. J Allergy Clin Immunol 2004;114:561-7.

28. Rachmiel M, Waisel Y, Verliger H, Keynan N, Katz Y. [Correlation between exposure to allergenic pollens and allergic manifestations]. Harefuah 1996;130:505-11, 584.

29. Vartiainen E, Petäys T, Haahtela T, Jousilahti P, Pekkanen J. Allergic diseases, skin prick test responses, and IgE levels in North Karelia, Finland, and the Republic of Karelia, Russia. J Allergy Clin Immunol 2002;109: 643-8.

30. Asher MI, Keil U, Anderson HR, et al. International Study of Asthma and Allergies in Childhood (ISAAC): rationale and methods. Eur Respir J 1995;8:483-91. 